\title{
Fractional calculus model of GATA-switching for regulating the differentiation of a hematopoietic stem cell
}

\author{
Ahmed Alsaedi ${ }^{*}$, Alexey Zaikin ${ }^{1,2}$, Bashir Ahmad ${ }^{1}$, Fuad Alsaadi ${ }^{3}$ and Moustafa El-Shahed ${ }^{1,4}$
}

"Correspondence:

aalsaedi@hotmail.com

${ }^{1}$ Nonlinear Analysis and Applied

Mathematics Research Group

(NAAM), Department of

Mathematics, Faculty of Science,

King Abdulaziz University,

P.O. Box 80257, Jeddah, 21589,

Saudi Arabia

Full list of author information is

available at the end of the article

\begin{abstract}
This paper deals with the fractional order model for GATA-switching for regulating the differentiation of a hematopoietic stem cell. We give a detailed analysis for the asymptotic stability of the model. The Adams-Bashforth-Moulton algorithm has been used to solve and simulate the system of differential equations.
\end{abstract}

Keywords: GATA-switching; fractional order dynamic system; stability; numerical methods

\section{Introduction}

Hematopoiesis is a highly orchestrated developmental process that comprises various developmental stages of hematopoietic stem cells (HSCs). During development, the decision to leave the self-renewing state and selection of a differentiation pathway is regulated by a number of transcription factors. Among them, genes GATA-1 and PU.1 form a core negative feedback module to regulate the genetic switching between the cell fate choices of HSCs. The transcription factors PU.1 and GATA-1 are known to be important in the development of blood progenitor cells. Specifically they are thought to regulate the differentiation of progenitor cells into the granulocyte/macrophage lineage and the erythrocyte/megakaryocite lineage. Although extensive experimental studies have revealed the mechanisms to regulate the expression of these two genes, it is still unclear how this simple module regulates the genetic switching $[1,2]$.

The notion of fractional calculus was anticipated by Leibniz, one of the founders of standard calculus, in a letter written in 1695 . Recently great considerations have been made to the models of FDEs in different areas of research. The most essential property of these models is their non-local property which does not exist in the integer order differential operators. We mean by this property that the next state of a model depends not only upon its current state but also upon all of its historical states [3-9].

In this paper, we consider the fractional model for GATA-switching for regulating the differentiation of a hematopoietic stem cell. We give a detailed analysis for the asymptotic stability of the model. The Adams-Bashforth-Moulton algorithm has been used to solve and simulate the system of differential equations.

@2014 Alsaedi et al.; licensee Springer. This is an Open Access article distributed under the terms of the Creative Commons Attribution License (http://creativecommons.org/licenses/by/2.0), which permits unrestricted use, distribution, and reproduction in any medium, provided the original work is properly cited. 


\section{Description of the model}

In [2], Tian and Smith-Miles proposed a mathematical model for the GATA-PU.1 regulatory network including genes GATA-1, GATA-2 and PU.1. Under some assumptions, they proposed the model to realize the genetic switching of the GATA-PU.1 regulatory network of the following form:

$$
\begin{aligned}
& \frac{d x}{d t}=\frac{a_{1} x+a_{2} y}{a_{3}+a_{4} x+a_{5} y+a_{6} z+a_{7} x z}-k_{1} x, \\
& \frac{d y}{d t}=\frac{b_{1} y}{b_{2}+b_{3} x+b_{4} y+b_{5} z+b_{6} y z}-k_{2} y, \\
& \frac{d z}{d t}=\frac{c_{1} z}{c_{2}+c_{3} x+c_{4} y+c_{5} z+c_{6} x z+c_{7} y z}-k_{3} z,
\end{aligned}
$$

where $x, y$ and $z$ are the concentrations of TFs GATA-1, GATA-2 and PU.1, respectively, $a_{1}, b_{1}$ and $c_{1}$ represent the expression rates of genes GATA-1, GATA-2 and PU.1 autoregulated by itself, respectively, $a_{2}$ is the expression rate of gene GATA-1 regulated by TF GATA-2, $k_{1}, k_{2}$ and $k_{3}$ are the degradation rates of TFs GATA-1, GATA-2 and PU.1, respectively. There are 23 rate constants in the proposed mathematical model (2.1). Now we introduce fractional order into the ODE model by (2.1). The new system is described by the following set of fractional order differential equations:

$$
\begin{aligned}
& D_{t}^{\alpha} x=\frac{a_{1} x+a_{2} y}{a_{3}+a_{4} x+a_{5} y+a_{6} z+a_{7} x z}-k_{1} x, \\
& D_{t}^{\alpha} y=\frac{b_{1} y}{b_{2}+b_{3} x+b_{4} y+b_{5} z+b_{6} y z}-k_{2} y, \\
& D_{t}^{\alpha} z=\frac{c_{1} z}{c_{2}+c_{3} x+c_{4} y+c_{5} z+c_{6} x z+c_{7} y z}-k_{3} z,
\end{aligned}
$$

where $\alpha$ is a parameter describing the order of the fractional time derivative in the Caputo sense defined as

$$
D_{t}^{\alpha} f(t)=\frac{1}{\Gamma(n-\alpha)} \int_{0}^{t} \frac{f^{(n)}(s)}{(t-s)^{\alpha-n+1}} d s, \quad n=[\alpha]+1 .
$$

\section{Equilibrium points and stability}

In the following, we discuss the stability of the commensurate fractional ordered dynamical system

$$
D_{t}^{\alpha} x_{i}=f_{i}\left(x_{1}, x_{2}, x_{3}\right), \quad \alpha \in(0,1), 1 \leq i \leq 3
$$

Let $E=\left(x_{1}^{*}, x_{2}^{*}, x_{3}^{*}\right)$ be an equilibrium point of system (3.1) and $x_{i}=x_{i}^{*}+\eta_{i}$, where $\eta_{i}$ is a small disturbance from a fixed point. Then

$$
\begin{aligned}
D_{t}^{\alpha} \eta_{i} & =D_{t}^{\alpha} x_{i} \\
& =f_{i}\left(x_{1}^{*}+\eta_{1}, x_{2}^{*}+\eta_{2}, x_{3}^{*}+\eta_{3}\right) \\
& \approx \eta_{1} \frac{\partial f_{i}(E)}{\partial x_{1}}+\eta_{2} \frac{\partial f_{i}(E)}{\partial x_{2}}+\eta_{3} \frac{\partial f_{i}(E)}{\partial x_{3}} .
\end{aligned}
$$


System (3.2) can be written as

$$
D_{t}^{\alpha} \eta=J \eta
$$

where $\eta=\left(\eta_{1}, \eta_{2}, \eta_{3}\right)^{T}$ and $J$ is the Jacobian matrix evaluated at the equilibrium points. Using Matignon's results [10], it follows that the linear autonomous system (3.3) is asymptotically stable if $|\arg (\lambda)|>\frac{\alpha \pi}{2}$ is satisfied for all eigenvalues of matrix $J$ at the equilibrium point $E=\left(x_{1}^{*}, x_{2}^{*}, x_{3}^{*}\right)$.

If $p(x)=x^{3}+a_{1} x^{2}+a_{2} x+a_{3}$, let $D(p)$ denote the discriminant of a polynomial $p(x)$, then

$$
D(p)=-\left|\begin{array}{ccccc}
1 & a_{1} & a_{2} & a_{3} & 0 \\
0 & 1 & a_{1} & a_{2} & a_{3} \\
3 & 2 a_{1} & a_{2} & 0 & 0 \\
0 & 3 & a_{1} & a_{2} & 0 \\
0 & 0 & 3 & 2 a_{1} & a_{2}
\end{array}\right|=18 a_{1} a_{2} a_{3}+\left(a_{1} a_{2}\right)^{2}-4 a_{3} a_{1}^{3}-4 a_{2}^{3}-27 a_{3}^{2} .
$$

Following $[4,10-13]$, we have the proposition.

Proposition One assumes that $E_{1}$ exists in $R_{+}^{3}$.

(i) If the discriminant of $p(x), D(p)$ is positive and the Routh-Hurwitz conditions are satisfied, that is, $D(p)>0, a_{1}>0, a_{3}>0, a_{1} a_{2}>a_{3}$, then $E_{1}$ is locally asymptotically stable.

(ii) If $D(p)<0, a_{1}>0, a_{2}>0, a_{1} a_{2}=a_{3}, \alpha \in[0,1)$, then $E_{1}$ is locally asymptotically stable.

(iii) If $D(p)<0, a_{1}<0, a_{2}<0, \alpha>\frac{2}{3}$, then $E_{1}$ is unstable.

(iv) The necessary condition for the equilibrium point $E_{1}$ to be locally asymptotically stable is $a_{3}>0$.

One can verify that system (2.2) has the following three steady states:

$$
E_{0}=(0,0,0), \quad E_{1}=\left(\frac{a_{1}-k_{1} a_{3}}{k_{1} a_{3}}, 0,0\right), \quad E_{3}=\left(0,0, \frac{c_{1}-k_{3} c_{2}}{k_{3} c_{5}}\right) .
$$

Theorem 3.1 The trivial steady state $E_{0}$ is locally asymptotically stable if the following conditions are satisfied: $\frac{a_{1}}{a_{3}}<k_{1}, \frac{b_{1}}{b_{2}}<k_{2}, \frac{c_{1}}{c_{2}}<k_{3}$.

Proof The trivial steady state $E_{0}$ is locally asymptotically stable if all the eigenvalues $\lambda_{i}$, $i=1,2,3$, of the Jacobian matrix $J\left(E_{0}\right)$ satisfy the following condition $[9,14-16]$ :

$$
\left|\arg \left(\lambda_{i}\right)\right|>\frac{\alpha \pi}{2} .
$$

The Jacobian matrix $J\left(E_{0}\right)$ for the system given in (2.2) evaluated at the steady state $E_{0}$ is as follows:

$$
J\left(E_{0}\right)=\left(\begin{array}{ccc}
\frac{a_{1}}{a_{3}}-k_{1} & \frac{a_{2}}{a_{3}} & 0 \\
0 & \frac{b_{1}}{b_{2}}-k_{2} & 0 \\
0 & 0 & \frac{c_{1}}{c_{2}}-k_{3}
\end{array}\right) .
$$

The eigenvalues of the Jacobian matrix $J\left(E_{0}\right)$ are $\lambda_{1}=\frac{a_{1}}{a_{3}}-k_{1}, \lambda_{2}=\frac{b_{1}}{b_{2}}-k_{2}, \lambda_{3}=\frac{c_{1}}{c_{2}}-k_{3}$. 
Hence $E_{0}$ is locally asymptotically stable if the following conditions are satisfied: $\frac{a_{1}}{a_{3}}<k_{1}$, $\frac{b_{1}}{b_{2}}<k_{2}, \frac{c_{1}}{c_{2}}<k_{3}$.

Theorem 3.2 The steady state $E_{1}$ with high expression level of gene GATA-1 is stable if the following conditions are satisfied:

$$
\frac{a_{3} k_{1}}{a_{1}}<1, \quad \frac{a_{4} k_{1} b_{1}}{a_{4} k_{1} b_{2}+b_{3}\left(a_{1}-a_{3} k_{1}\right)}<k_{2}, \quad \frac{a_{4} k_{1} c_{1}}{a_{4} k_{1} c_{2}+c_{3}\left(a_{1}-a_{3} k_{1}\right)}<k_{3} .
$$

Proof The Jacobian matrix of nonlinear system (2.2) for this steady state $E_{1}$ is

$$
J\left(E_{1}\right)=\left(\begin{array}{ccc}
k_{1}\left(\frac{a_{3} k_{1}-a_{1}}{a_{1}}\right) & \frac{k_{1}\left(a_{2} a_{4}+a_{5}\left(-a_{1}+a_{3} k_{1}\right)\right)}{a_{1} a_{4}} & -\frac{\left(a_{1}-a_{3} k_{1}\right)\left(a_{4} a_{6} k_{1}+a_{7}\left(a_{1}-a_{3} k_{1}\right)\right)}{a_{1} a_{4}^{2}} \\
0 & \frac{a_{4} k_{1} b_{1}}{a_{4} k_{1} b_{2}+b_{3}\left(a_{1}-a_{3} k_{1}\right)}-k_{2} & 0 \\
0 & 0 & \frac{a_{4} k_{1} c_{1}}{a_{4} k_{1} c_{2}+c_{3}\left(a_{1}-a_{3} k_{1}\right)}-k_{3}
\end{array}\right) .
$$

The three eigenvalues of the Jacobian matrix are:

$$
\begin{aligned}
& \lambda_{1}=k_{1}\left(\frac{a_{3} k_{1}-a_{1}}{a_{1}}\right), \\
& \lambda_{2}=\frac{a_{4} k_{1} b_{1}}{a_{4} k_{1} b_{2}+b_{3}\left(a_{1}-a_{3} k_{1}\right)}-k_{2}, \\
& \lambda_{3}=\frac{a_{4} k_{1} c_{1}}{a_{4} k_{1} c_{2}+c_{3}\left(a_{1}-a_{3} k_{1}\right)}-k_{3} .
\end{aligned}
$$

Theorem 1 of [2] has the same results for the integer order model (and has some misprints in the first condition). They claimed that $\lambda_{1}$ is negative, but the sign of $\lambda_{1}$ depends on the quantity $\left(\frac{a_{3} k_{1}-a_{1}}{a_{1}}\right)$.

Theorem 3.3 The steady state $E_{3}$ with high expression level of gene PU.1 is stable if the following conditions are satisfied:

$$
\frac{c_{2} k_{3}}{c_{1}}<1, \quad \frac{a_{1} c_{5} k_{3}}{a_{3} c_{5} k_{3}+a_{6}\left(c_{1}-c_{2} k_{3}\right)}<k_{1}, \quad \frac{b_{1} c_{5} k_{3}}{c_{5} k_{3} b_{2}+b_{5}\left(c_{1}-c_{2} k_{3}\right)}<k_{2} .
$$

Proof The Jacobian matrix of nonlinear system (2.1) for this steady state $E_{3}$ is

$$
J\left(E_{3}\right)=\left(\begin{array}{c}
\frac{a_{1} c_{5} k_{3}}{a_{3} c_{5} k_{3}+a_{6}\left(c_{1}-c_{2} k_{3}\right)}-k_{1} \\
0 \\
-\frac{\left(c_{1}-c_{2} k_{3}\right)\left(c_{3} c_{5} k_{3}+c_{6}\left(c_{1}-c_{2} k_{3}\right)\right)}{c_{1} c_{5}^{2}}
\end{array}\right.
$$

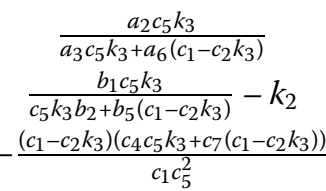

$$
\left.\begin{array}{c}
0 \\
0 \\
k_{3}\left(\frac{c_{2} k_{3}-c_{1}}{c_{1}}\right)
\end{array}\right)
$$

The three eigenvalues of the Jacobian matrix are:

$$
\begin{aligned}
& \lambda_{1}=k_{3}\left(\frac{c_{2} k_{3}}{c_{1}}-1\right), \\
& \lambda_{2}=\frac{a_{1} c_{5} k_{3}}{a_{3} c_{5} k_{3}+a_{6}\left(c_{1}-c_{2} k_{3}\right)}-k_{1}, \\
& \lambda_{3}=\frac{b_{1} c_{5} k_{3}}{c_{5} k_{3} b_{2}+b_{5}\left(c_{1}-c_{2} k_{3}\right)}-k_{2} .
\end{aligned}
$$


Table 1 The equilibrium points and the eigenvalues of the system

\begin{tabular}{ll}
\hline Equilibrium point & Eigenvalues \\
\hline$E_{4}=(659.2192685,0,0)$ & $\{-0.909177,0.435225,-0.234831\}$ \\
$E_{5}=(322.17148,1.36478,0)$ & $\{-0.909177,0.435225,-0.234831\}$ \\
$E_{6}=(3.812275,13.9889,0)$ & $\{-1.37375,0.948121,-0.560537\}$ \\
$E_{7}=\left(15.99,2.19 \times 10^{-12}, 1.153\right)$ & $\{26.522544,-0.929339,0.0585563\}$ \\
$E_{8}=(3.6329,13.065,1.17301)$ & $\{-1.38529,-0.591193,-0.203561\}$ \\
$E_{9}=(0.00054,0.0042,241.024)$ & $\{-0.622622,-0.345295,0.0472589\}$ \\
\hline
\end{tabular}

Under the conditions of Theorem 3.3, we can conclude that steady state $E_{3}$ with high expression level of gene PU.1 is stable.

Following [2], parameters of the model are as follows:

$$
\begin{aligned}
& \left(a_{1}, a_{2}, a_{3}, a_{4}, a_{5}, a_{6}, a_{7}\right)=(731.7409,856.1247,1,1.6,398.9719,44.8982,53.0), \\
& \left(b_{1}, b_{2}, b_{3}, b_{4}, b_{5}, b_{6}\right)=(18470.6419,1,37.3615,942.1939,55.0375,53.0), \\
& \left(c_{1}, c_{2}, c_{3}, c_{4}, c_{5}, c_{6}, c_{7}\right)=(12391.1968,1,710.4490,522.4385,170.0,1700.0,1700.0)
\end{aligned}
$$

and

$$
\left(k_{1}, k_{2}, k_{3}\right)=(0.6931,1.3863,0.2888)
$$

Using these parameters, one can verify that the system has six nontrivial equilibrium points. The equilibrium points and the eigenvalues of corresponding Jacobian matrix are given in Table 1.

It is clear from the table, that the equilibrium point $E_{8}$ is a stable point. The other points are unstable.

\section{Numerical methods and simulations}

Since most of the fractional-order differential equations do not have exact analytic solutions, approximation and numerical techniques must be used. Several analytical and numerical methods have been proposed to solve the fractional-order differential equations. For numerical solutions of system (2.2), one can use the generalized Adams-BashforthMoulton method. To give the approximate solution by means of this algorithm, consider the following nonlinear fractional differential equation [17]:

$$
\begin{aligned}
& D_{t}^{\alpha} y(t)=f(t, y(t)), \quad 0 \leq t \leq T, \\
& y^{(k)}(0)=y_{0}^{k}, \quad k=0,1,2, \ldots, m-1, \text { where } m=[\alpha] .
\end{aligned}
$$

This equation is equivalent to the Volterra integral equation

$$
y(t)=\sum_{k=0}^{m-1} y_{0}^{(k)} \frac{t^{k}}{k !}+\frac{1}{\Gamma(\alpha)} \int_{0}^{t}(t-s)^{\alpha-1} f(s, y(s)) d s
$$

Diethelm et al. used the predictor-correctors scheme [11, 12] based on the Adams-Bashforth-Moulton algorithm to integrate Eq. (4.1). By applying this scheme to the fractionalorder model GATA-switching for regulating the differentiation of a hematopoietic stem 
cell, and setting $h=\frac{T}{N}, t_{n}=n h, n=0,1,2, \ldots, N \in Z^{+}$, Eq. (4.1) can be discretized as follows [17]:

$$
\begin{aligned}
x_{n+1}= & x_{0}+\frac{h^{\alpha}}{\Gamma(\alpha+2)}\left(\frac{a_{1} x_{n+1}^{p}+a_{2} y_{n+1}^{p}}{a_{3}+a_{4} x_{n+1}^{p}+a_{5} y_{n+1}^{p}+a_{6} z_{n+1}^{p}+a_{7} x_{n+1}^{p} z_{n+1}^{p}}-k_{1} x_{n+1}^{p}\right) \\
& +\frac{h^{\alpha}}{\Gamma(\alpha+2)} \sum_{j=0}^{n} a_{j, n+1}\left(\frac{a_{1} x_{j}+a_{2} y_{j}}{a_{3}+a_{4} x_{j}+a_{5} y_{j}+a_{6} z_{j}+a_{7} x_{j} z_{j}}-k_{1} x_{j}\right), \\
y_{n+1}= & y_{0}+\frac{h^{\alpha}}{\Gamma(\alpha+2)}\left(\frac{b_{1} y_{n+1}^{p}}{b_{2}+b_{3} x_{n+1}^{p}+b_{4} y_{n+1}^{p}+b_{5} z_{n+1}^{p}+b_{6} y_{n+1}^{p} z_{n+1}^{p}}-k_{2} y_{n+1}^{p}\right) \\
& +\frac{h^{\alpha}}{\Gamma(\alpha+2)} \sum_{j=0}^{n} a_{j, n+1}\left(\frac{b_{1} y_{j}}{b_{2}+b_{3} x_{j}+b_{4} y_{j}+b_{5} z_{j}+b_{6} y_{j} z_{j}}-k_{2} y_{j}\right), \\
z_{n+1}= & z_{0}+\frac{h^{\alpha}}{\Gamma(\alpha+2)}\left(\frac{c_{1} z_{n+1}^{p}}{c_{2}+c_{3} x_{n+1}^{p}+c_{4} y_{n+1}^{p}+c_{5} z_{n+1}^{p}+c_{6} x_{n+1}^{p} z_{n+1}^{p}+c_{7} y_{n+1}^{p} z_{n+1}^{p}}-k_{3} z_{n+1}^{p}\right) \\
& +\frac{h^{\alpha}}{\Gamma(\alpha+2)} \sum_{j=0}^{n} a_{j, n+1}\left(\frac{c_{1} z_{j}}{c_{2}+c_{3} x_{j}+c_{4} y_{j}+c_{5} z_{j}+c_{6} x_{j} z_{j}+c_{7} y_{j} z_{j}}-k_{3} z_{j}\right),
\end{aligned}
$$

where

$$
\begin{aligned}
& x_{n+1}^{p}=x_{0}+\frac{1}{\Gamma(\alpha)} \sum_{j=0}^{n} b_{j, n+1}\left(\frac{a_{1} x_{j}+a_{2} y_{j}}{a_{3}+a_{4} x_{j}+a_{5} y_{j}+a_{6} z_{j}+a_{7} x_{j} z_{j}}-k_{1} x_{j}\right), \\
& y_{n+1}^{p}=y_{0}+\frac{1}{\Gamma(\alpha)} \sum_{j=0}^{n} b_{j, n+1}\left(\frac{b_{1} y_{j}}{b_{2}+b_{3} x_{j}+b_{4} y_{j}+b_{5} z_{j}+b_{6} y_{j} z_{j}}-k_{2} y_{j}\right), \\
& z_{n+1}^{p}=z_{0}+\frac{1}{\Gamma(\alpha)} \sum_{j=0}^{n} b_{j, n+1}\left(\frac{c_{1} z_{j}}{c_{2}+c_{3} x_{j}+c_{4} y_{j}+c_{5} z_{j}+c_{6} x_{j} z_{j}+c_{7} y_{j} z_{j}}-k_{3} z_{j}\right), \\
& a_{j, n+1}= \begin{cases}n^{\alpha+1}-(n-\alpha)(n+1), & j=0, \\
(n-j+2)^{\alpha+1}+(n-j)^{\alpha+1}-2(n-j+1)^{\alpha+1}, & 1 \leq j \leq n, \\
1, & j=n+1,\end{cases} \\
& b_{j, n+1}=\frac{h^{\alpha}}{\alpha}\left((n-j+1)^{\alpha}-(n-j)^{\alpha}\right), \quad 0 \leq j \leq n .
\end{aligned}
$$

Figure 1 illustrates the distribution of the concentration GATA-1, GATA-2 and PU.1 with time. It is observed that GATA-1 is increasing with time and reaches its equilibrium point (3.6329), while PU.1 seems to decrease with time and reaches its steady state (1.17301). On the other hand, the GATA-2 gene seems to decrease with time and reach its equilibrium point (13.065). Figure 2 indicates the behavior of the approximate solutions for system (2.2) obtained for the values of $\alpha=0.6$. In Figure 3, the variation of GATA-1 vs. time $t$ is shown for different values of $\alpha=1,0.6$ by fixing other parameters. Figure 4 depicts GATA- 2 vs. time $t$. Figure 4 shows similar variations of GATA- 1 with various values of $\alpha$. In Figure 5, the variation of PU.1 vs. time $t$ is shown for different values of $\alpha$ that increase, $\alpha$ decreases with the concentration of PU.1 gene. 


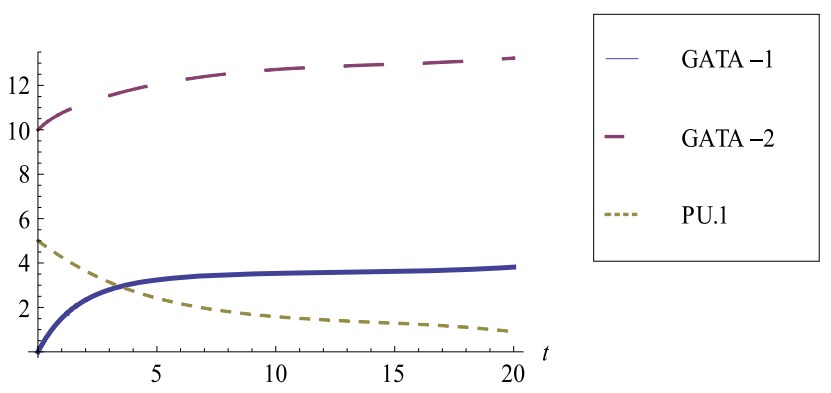

Figure 1 Variation of GATA-1, GATA-2 and PU. 1 with time for $\alpha=1$.
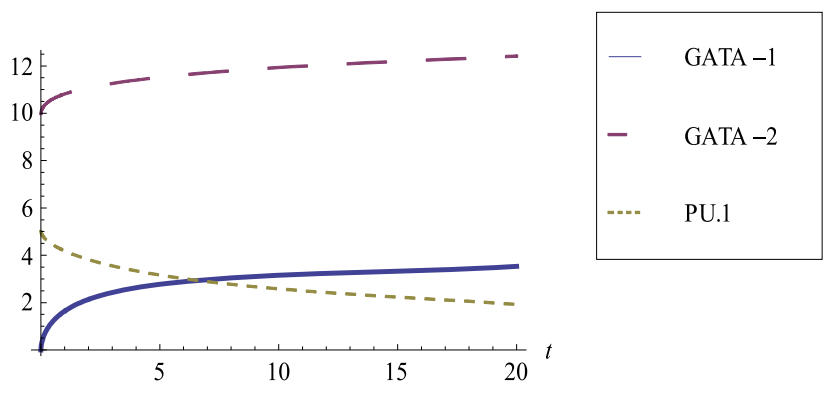

Figure 2 Variation of GATA-1, GATA-2 and PU. 1 with time for $\alpha=0.6$.

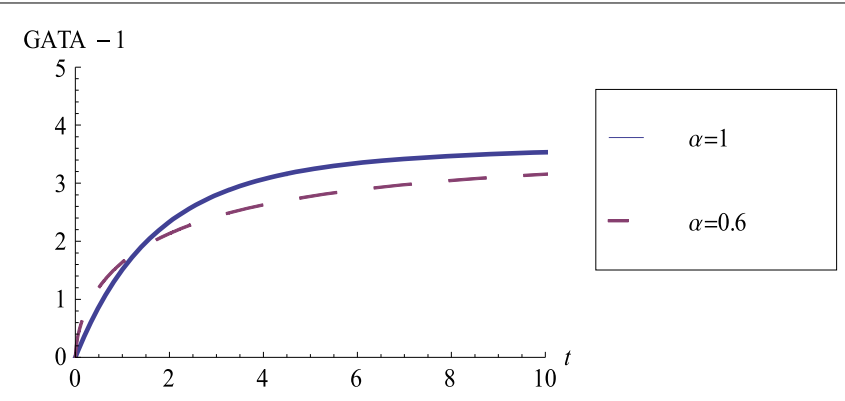

Figure 3 Variation of GATA-1 with time for different values of $\alpha$.

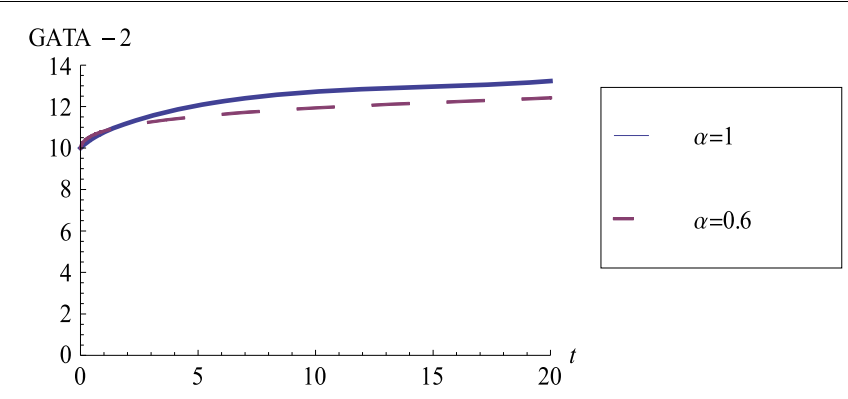

Figure 4 Variation of GATA-2 with time for different values of $\alpha$. 


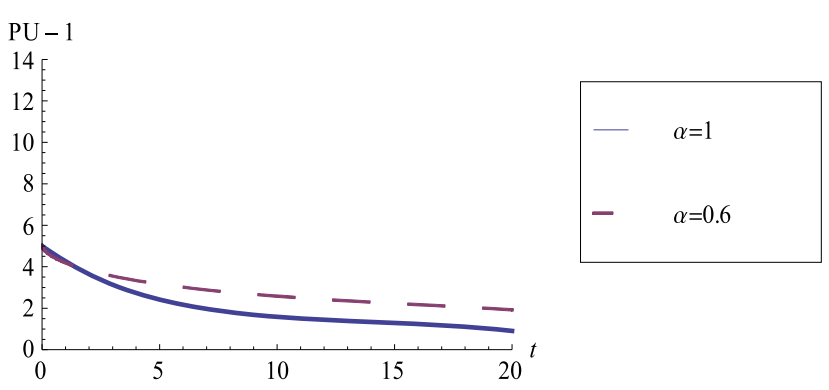

Figure 5 Variation of PU.1 with time for different values of $\alpha$.

\section{Conclusions}

In this paper, we consider the fractional model for GATA-switching for regulating the differentiation of a hematopoietic stem cell. We have obtained a stability condition for equilibrium points. We have also given a numerical example and verified our results. One should note that although the equilibrium points are the same for both integer order and fractional order models, the solution of the fractional order model tends to the fixed point over a longer period of time. One also needs to mention that when dealing with real life problems, the order of the system can be determined by using the collected data.

Competing interests

The authors declare that they have no competing interests.

\section{Authors' contributions}

All authors contributed equally to the writing of this paper. All authors read and approved the final manuscript.

\section{Author details}

${ }^{1}$ Nonlinear Analysis and Applied Mathematics Research Group (NAAM), Department of Mathematics, Faculty of Science, King Abdulaziz University, P.O. Box 80257, Jeddah, 21589, Saudi Arabia. ${ }^{2}$ Institute of Women's Health, Department of Mathematics, University College London, Gower Street, London, WC1E 6BT, United Kingdom. ${ }^{3}$ Department of Electrical and Computer Engineering, Faculty of Engineering, King Abdulaziz University, P.O. Box 80204, Jeddah, 21589, Saudi Arabia. ${ }^{4}$ Department of Mathematics, Faculty of Art and Sciences, Qassim University, P.O. Box 3771, Unizah, Qassim, Saudi Arabia.

Received: 16 March 2014 Accepted: 14 May 2014 Published: 24 Jul 2014

\section{References}

1. Roeder, I, Glauche, I: Towards an understanding of lineage specification in hematopoietic stem cells: a mathematical model for the interaction of transcription factors GATA-1 and PU.1. J. Theor. Biol. 241, 852-865 (2006)

2. Tian, T, Smith-Miles, K: Mathematical modeling of GATA-switching for regulating the differentiation of hematopoietic stem cell. BMC Syst. Biol. 8(suppl. 1), S8 (2014)

3. Baleanu, D, Diethelm, K, Scalas, E, Trujillo, JJ: Fractional Calculus Models and Numerical Methods. World Scientific, Singapore (2012)

4. Ding, Y, Ye, H: A fractional-order differential equation model of HIV infection of CD4 ${ }^{+} \mathrm{T}$-cells. Math. Comput. Model. 50, 386-392 (2009)

5. Golmankhaneh, AK, Arefi, R, Baleanu, D: Synchronization in a nonidentical fractional order of a proposed modified system. J. Vib. Control 24, 1077546313494953 (2013)

6. Golmankhaneh, AK, Arefi, R, Baleanu, D: The proposed modified Liu system with fractional order. Adv. Math. Phys. 2013, 186037 (2013)

7. Kilbas, AA, Srivastava, HM, Trujillo, JJ: Theory and Application Fractional Differential Equations. Elsevier, Amsterdam (2006)

8. Podlubny, I: Fractional Differential Equations. Academic Press, New York (1999)

9. Ye, H, Ding, Y: Nonlinear dynamics and chaos in a fractional-order HIV model. Math. Probl. Eng. 2009, Article ID 378614 (2009)

10. Matignon, D: Stability results for fractional differential equations with applications to control processing. In: Computational Engineering in Systems Applications, Lille, France, vol. 2, p. 963 (1996)

11. Diethelm, K, Ford, NJ: Analysis of fractional differential equations. J. Math. Anal. Appl. 265, 229-248 (2002)

12. Diethelm, K, Ford, NJ, Freed, AD: A predictor-corrector approach for the numerical solution of fractional differential equations. Nonlinear Dyn. 29, 3-22 (2002)

13. Ozalp, N, Demirci, E: A fractional order SEIR model with vertical transmission. Math. Comput. Model. 54, 1-6 (2011)

14. Ahmed, E, El-Sayed, AMA, El-Mesiry, EM, El-Saka, HAA: Numerical solution for the fractional replicator equation. Int. J. Mod. Phys. C 16, 1-9 (2005) 
15. Ahmed, E, El-Sayed, AMA, El-Saka, HAA: On some Routh-Hurwitz conditions for fractional order differential equations and their applications in Lorenz, Rossler, Chua and Chen systems. Phys. Lett. A 358, 1-4 (2006)

16. Ahmed, E, El-Sayed, AMA, El-Saka, HAA: Equilibrium points, stability and numerical solutions of fractional-order predator-prey and rabies models. J. Math. Anal. Appl. 325, 542-553 (2007)

17. Li, C, Tao, C: On the fractional Adams method. Comput. Math. Appl. 58, 1573-1588 (2009)

10.1186/1687-1847-2014-201

Cite this article as: Alsaedi et al.: Fractional calculus model of GATA-switching for regulating the differentiation of a hematopoietic stem cell. Advances in Difference Equations 2014, 2014:201

Submit your manuscript to a SpringerOpen ${ }^{\circ}$ journal and benefit from:

- Convenient online submission

- Rigorous peer review

- Immediate publication on acceptance

- Open access: articles freely available online

- High visibility within the field

- Retaining the copyright to your article

Submit your next manuscript at $>$ springeropen.com 